\title{
Apresentação Pélvica na Gestação de Termo em Pacientes com Partos Vaginais Prévios
}

\author{
Breech Presentation in Term Pregnancy in Patients with Previous Vaginal Deliveries
}

\begin{abstract}
José Mauro Madi, Renato Luís Rombaldi, Edson Nunes de Morais, Breno Fauth de Araújo,
\end{abstract} Sônia Regina Cabral Madi, Dilma Tonoli Tessari, Maíra Zapparoli

\section{RESUM0}

Objetivo: avaliar os resultados obstétricos e perinatais em casos de fetos em apresentação pélvica, de termo, nascidos de pacientes com partos vaginais prévios, comparando-os a fetos de termo, em apresentação cefálica.

Pacientes e Métodos: foram analisados retrospectivamente 8.350 nascimentos ocorridos no periodo de março de 1998 a julho de 2003. Ocorreram 419 partos (5,1\%) em apresentação pélvica, dos quais selecionaram-se 58 casos (grupo pélvico), que deveriam ter as seguintes caracteristicas: antecedentes de um ou mais filhos nascidos pela via transpélvica, idade gestacional igual ou superior a 37 semanas, ausência de malformações fetais, inexistência de intercorrências durante a gestação, peso do recém-nascido no nascimento igual ou superior a $2.500 \mathrm{~g}$ e inferior a $3.750 \mathrm{~g}$, e sem cesárea anterior. Esse grupo foi comparado a outro formado por 1.327 fetos com características semelhantes, em apresentação cefálica, de gestantes sem cesárea prévia (grupo cefálico). Analisaram-se a idade materna, paridade, idade gestacional, via de parto, peso do recém-nascido, presença de mecônio, índice de Apgar no primeiro e quinto minutos, necessidade de internação na unidade de tratamento intensivo neonatal e ocorrência de recém-nascidos pequenos e grandes para a idade gestacional. Os dados obstétricos e perinatais foram analisados pelo $\chi^{2}$ e teste t de Student. Considerou-se como significante $p<0,05$.

Resultados: comparados os grupos pélvico e cefálico, respectivamente, mostraram-se

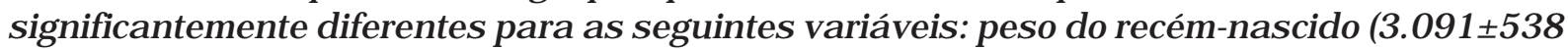
vs $3.250 \pm 497 \mathrm{~g} ; p<0,01)$, parto vaginal (63,8 vs $95,0 \%$; $p<0,0001)$, cesárea $(36,2$ vs $5,0 \%$; $p<0,0001)$ e índice de Apgar $<4$ e $<7$ no primeiro minuto ( $p<0,0001)$.

Conclusões: os resultados permitem concluir que em fetos de termo, em apresentação pélvica, de gestantes com partos vaginais prévios, o peso fetal, a via de parto e os índices de Apgar de primeiro minuto são diferentes, se comparados a fetos em apresentação cefálica, de gestantes com as mesmas características.

PALAVRAS-CHAVE: Apresentação pélvica. Morbidade perinatal. Parto vaginal. Cesárea.

\section{Introdução}

A apresentação pélvica ocorre quando o feto,

Unidade de Medicina Perinatal do Hospital Geral da Universidade de Caxias do Sul.

Correspondência:

José Mauro Madi

Rua Alfredo Chaves, 705/804 - Centro

95020-460 - Caxias do Sul - RS

e-mail: madi@visao.com.br disposto longitudinalmente dentro do útero, está com o polo pélvico situado na área do estreito superior $^{1}$, a incidência é de 3,0 a 4,0\% das gestações a termo. Nos últimos 20 anos, em virtude do aumento global das taxas de operação cesariana, bem como da ampliação das indicações, alguns autores têm referido melhores condições de nascimento para fetos em apresentação pélvica ${ }^{2}$. Hannah et al. $^{3}$, em 2000, mostraram em estudo 
multicêntrico que a cesárea eletiva é melhor que o parto vaginal para fetos de termo em apresentação pélvica. Por outro lado, as complicações maternas não diferiram entre os dois grupos estudados, cesárea ou parto vaginal.

$\mathrm{Na}$ tentativa de se lograr o parto pela via transpélvica, vários são os fatores que devem ser considerados na seleção adequada das parturientes, incluindo-se a paridade, bacia óssea materna, atividade uterina, boa prensa abdominal, correta identificação da idade gestacional e peso fetal, grau de flexão e rotação da cabeça fetal, variedade de apresentação, integridade das membranas ovulares, presença de cicatriz uterina e de circulares de cordão ${ }^{4}$. Em situações em que esses parâmetros não puderem ser adequadamente avaliados, o risco de óbito fetal, prolapso de cordão umbilical, traumas, baixos indices de Apgar ao nascimento e morte neonatal tendem a aumentar significativamente ${ }^{4}$.

Provavelmente, esse é o motivo pelo qual a operação cesariana tem tido indicação quase que rotineira nas apresentações pélvicas, modo de nádegas e suas variações, embora não existam conclusões definitivas sobre a melhor via de parto para essa apresentação ${ }^{4}$.

Lindqvist et al. ${ }^{5}$, estudando gestações únicas com apresentação pélvica, encontraram maior mortalidade perinatal quando o parto ocorria pela via transvaginal do que pela via abdominal. Em estudo controlado e randomizado, comparando a morbidade e a mortalidade destas duas vias de parto, não foi encontrada diferença significante entre as vias de parturição em gestações de termo para mortalidade perinatal e índice de Apgar no quinto minuto inferior a 7 , ainda que a cesariana estivesse associada a aumento da taxa de morbidade materna ${ }^{6}$.

Posto que persistem dúvidas quanto à melhor via para a assistência ao parto em apresentação pélvica, decidiu-se realizar este estudo que objetiva avaliar os resultados obstétricos e perinatais em casos de fetos em apresentação pélvica, de termo, em gestantes com partos vaginais anteriores.

\section{Pacientes e Métodos}

Estudo retrospectivo e descritivo de 8.350 nascimentos consecutivos ocorridos na Unidade de Medicina Perinatal do Hospital Geral de Caxias do Sul (UMP/HG), no período de março de 1998 a julho de 2003. A UMP/HG é serviço de referência para a região nordeste do RS, e que atende exclusivamente usuárias do Sistema Único de Saúde.
Do total de nascimentos no período, em 419 $(5,1 \%)$ o feto encontrava-se em apresentação pélvica. Desses, foram selecionados 58 casos $(13,8 \%)$, que preencheram os seguintes critérios de inclusão: gestante com partos vaginais prévios e sem cicatrizes de cesárea anterior, idade gestacional igual ou superior a 37 semanas e peso do recém-nascido no nascimento igual ou superior a 2.500 g e inferior a 3.750 g. Consideraramse como fatores de exclusão: gravidez múltipla, primiparidade, gestantes com parto anterior ocorrido pela via abdominal, idade gestacional inferior a 37 semanas, presença de intercorrências clínicas, óbito intra-uterino durante a atual gestação e malformação fetal.

Comparou-se o grupo pélvico a 1.327 casos em apresentação cefálica, oriundos de gestantes que não apresentavam cicatriz de cesárea prévia e que preenchiam os mesmos critérios de inclusão e exclusão do primeiro grupo, nascidos no mesmo período.

Foram analisadas variáveis maternas: idade (em anos); paridade; idade gestacional determinada por ocasião do parto, segundo o índice de Capurro; tempo de trabalho de parto analisado após a internação no UMP/HG; via de parto; variáveis perinatais: peso do recém-nascido; presença de mecônio no líquido amniótico; índice de Apgar no primeiro e quinto minuto; necessidade de internação na Unidade de Tratamento Intensivo Neonatal; ocorrência de pequenos e grandes para a idade gestacional, segundo Battaglia e Lubchenco ${ }^{7}$, mortalidade neonatal precoce (antes de 7 dias).

A apresentação pélvica na UMP/HG não constitui indicação obrigatória de parto cesáreo, já que a tentativa de parto vaginal é realizada para fetos com peso estimado entre $2.500 \mathrm{~g}$ e $3.700 \mathrm{~g}$ pela ultra-sonografia e palpação abdominal, em associação à adequada proporcionalidade da bacia pélvica materna.

Os dados obstétricos e perinatais foram inseridos no SPSS-PC 10.0 (Statistical Package for the Social Sciences for Personal Computer) e as avaliações estatísticas foram realizadas mediante a aplicação do teste não paramétrico do $\chi^{2}$ (via de parto, índice de Apgar, presença de mecônio, necessidade de tratamento em ambiente de intensivismo neonatal, mortalidade neonatal precoce, taxa de pequenos e grandes para a idade gestacional) e do teste $t$ de Student (idade materna, idade gestacional e paridade). Adotou-se o nível de significância de $5 \%$.

O projeto foi aprovado pela Comissão de Ética e Pesquisa da Universidade de Caxias do Sul. 


\section{Resultados}

Considerando-se os grupos de pélvicos e de cefálicos, a idade materna média foi de $30,9 \pm 6,5$ vs $29,5 \pm 6,2$ anos e a paridade média foi de $3,2 \pm 1,5$ vs 3,0 $\pm 1,4$ filhos, respectivamente; a idade gestacional por ocasião do parto foi de $39,5 \pm 1,4$ semanas em ambos os grupos. Esses parâmetros não apresentaram diferença significante. As variáveis citadas mostraram-se distribuídas de forma semelhante em ambos os grupos, caracterizando homogeneidade entre eles.

O tempo total de trabalho de parto observado após a internação em ambiente hospitalar foi de $185,3 \pm 144,1$ min para o grupo de pélvicos e $203,7 \pm 224,0$ min para o grupo de cefálicos. Esse dado não apresentou diferença significante.

No grupo dos pélvicos observou-se incidência de partos vaginais de $63,8 \%(n=37)$, contra $95 \%$ $(\mathrm{n}=1.261)$ do grupo dos cefálicos $(\mathrm{p}<0,0001)$.

O peso médio dos recém-nascidos por ocasião do nascimento foi de $3.091 \pm 538 \mathrm{~g}$ entre os pélvicos e $3.250 \pm 497 \mathrm{~g}$ entre os cefálicos, sendo essa diferença significante $(\mathrm{p}<0,01)$.

A distribuição dos índices de Apgar no primeiro e quinto minutos pode ser observada na Tabela 1. Houve diferença significante entre os valores de Apgar de primeiro minuto entre os grupos estudados, o mesmo não ocorrendo no quinto minuto. O percentual de recém-nascidos gravemente deprimidos no primeiro minuto entre os pélvicos foi aproximadamente seis vezes maior que entre os cefálicos.

Tabela 1 - Distribuição do índice de Apgar de $1^{\circ}$ e $5^{\circ}$ minuto dos recém-nascidos dos dois grupos estudados.

\begin{tabular}{|c|c|c|c|c|c|}
\hline \multirow[t]{2}{*}{ Índice de Apgar } & \multicolumn{2}{|c|}{ Pélvicos } & \multicolumn{2}{|c|}{ Cefálicos } & \multirow[t]{2}{*}{$p$} \\
\hline & $\mathrm{n}$ & $\%$ & $n$ & $\%$ & \\
\hline \multicolumn{6}{|l|}{$1^{\circ}$ minuto } \\
\hline$<4$ & 8 & 14,0 & 32 & 2,5 & $<0,0001$ \\
\hline $4-7$ & 10 & 17,5 & 71 & 5,5 & $<0,0001$ \\
\hline$\geq 7$ & 39 & 68,5 & 1.182 & 92,0 & $<0,0005$ \\
\hline \multicolumn{6}{|l|}{$5^{\circ}$ minuto } \\
\hline$<4$ & 1 & 1,7 & 6 & 0,5 & NS \\
\hline $4-7$ & 1 & 1,7 & 15 & 1,1 & NS \\
\hline$\geq 7$ & 56 & 96,6 & 1.295 & 98,4 & NS \\
\hline
\end{tabular}

As variáveis ocorrência de mecônio $(20,7$ vs $16,3 \%$ ), necessidade de tratamento intensivo neonatal $(12,1 \%$ vs $9,2 \%)$, mortalidade neonatal precoce $(1,7$ vs $2,9 \%)$, pequenos para a idade gestacional $(5,2$ vs $5,4 \%)$ e grandes para a idade gestacional (10,3 vs $14,2 \%)$ foram semelhantes entre os dois grupos (Tabela 2).

Tabela 2 - Distribuição dos resultados perinatais nos dois grupos estudados.

\begin{tabular}{lrrrrr}
\hline Variáveis & \multicolumn{2}{c}{ Pélvicos } & \multicolumn{2}{c}{ Cefálicos } & p \\
& $\mathbf{n}$ & \multicolumn{1}{c}{$\%$} & \multicolumn{1}{c}{$\mathbf{n}$} & \multicolumn{1}{c}{$\%$} & \\
\hline Presença de mecônio & 12 & 20,7 & 217 & 16,3 & NS \\
Necessidade de UTIN & 7 & 12,1 & 122 & 9,2 & NS \\
Mortalidade neonatal precoce* & 1 & 1,7 & 39 & 2,9 & NS \\
PIG & 3 & 5,2 & 71 & 5,4 & NS \\
GIG & 6 & 10,3 & 189 & 14,2 & NS \\
\hline
\end{tabular}

${ }^{*}<7$ dias *UTIN: Unidade de Tratamento Intensivo Neonatal; PIG: pequeno para a idade gestacional; GIG: grande para a idade gestacional.

\section{Discussão}

A via de parto, quando em apresentação pélvica, independente dos fatores tradicionalmente considerados e a despeito dos vários estudos e propostas publicadas na literatura atual, continua despertando discussão e motivando novas pesquisas. Por não haver proposta consensual a nortear o profissional da área, decidiu-se elaborar esse estudo, visando identificar fatores que possam, de alguma forma, trazer à luz novos dados a serem somados aos já existentes.

Para evitar os diferentes vieses característicos de estudo retrospectivo, foram selecionados somente os casos que incluíssem gestações de termo, cujo peso fetal na gestação em estudo estivesse compreendido entre 2.500 e $3.750 \mathrm{~g}$, sem malformações fetais e intercorrências clínicas, mas com partos vaginais prévios. O grupo controle, composto por fetos em apresentação cefálica, obedeceu aos mesmos critérios de inclusão e exclusão.

Ao se compararem os dois grupos, que se mostraram homogêneos, não se observou diferença significante entre as variáveis idade materna, paridade, idade gestacional por ocasião do parto e tempo de trabalho de parto. A mesma conclusão foi referida por Sobande et al. ${ }^{8}$. Ao contrário, Fawole et al. ${ }^{9}$, ao analisarem 266 partos pélvicos, referiram maior incidência em primigrávidas ${ }^{9}$.

Na UMP/HG, a incidência de mecônio na população geral por ocasião do parto varia em torno de $15,0 \%$. Sabe-se que o mecônio por si só não deve ser considerado representativo de comprometimento da higidez fetal, embora, quando intenso, espesso, possa se associar com a sindrome 
da aspiração de mecônio e alta morbimortalidade neonatal ${ }^{10-12}$. Nesse estudo, ainda que a incidência esteja levemente aumentada no grupo de fetos em apresentação pélvica, a sua presença não esteve associada ao tipo de apresentação fetal. Por outro lado, é de se supor que nos pélvicos, a presença de mecônio durante o trabalho de parto possa significar apenas a compressão do abdômen fetal quando de sua passagem pelo canal de parto. Convém enfatizar o fato de não terem sido incluídos no estudo os recém-nascidos pré-termo, de trato digestivo imaturo, em que a eliminação de mecônio não é característica ${ }^{12}$. Gonzalez de Dios et al. ${ }^{13}$ relataram incidência de $18 \%$ de mecônio ao considerar todos os partos de sua pesquisa.

O peso médio dos recém-nascidos observado no grupo de pélvicos esteve de acordo com os critérios utilizados na UMP/HG para o parto vaginal de fetos em apresentação pélvica. Permite-se a tentativa de parto pela via vaginal para conceptos com peso estimado entre 2.500 e $3.700 \mathrm{~g}$, desde que os critérios de factibilidade tenham sido cuidadosamente avaliados e confirmados; provavelmente esse fato tenha proporcionado maior número de partos vaginais. Houve diferença significante entre a média dos pesos e o tipo de parto. Sobande et al. ${ }^{8}$ não observaram diferença ao comparar o peso dos conceptos incluídos em seu estudo. Tunde-Byass e Hannah ${ }^{14}$ referiram que conceptos com peso estimado entre 2.500 e 4.000 g devem ser considerados de risco para resultados adversos.

Madi et al. ${ }^{15}$ descreveram incidência de cesárea no Hospital Geral de Caxias do Sul de $24 \%$. No presente estudo, essa incidência foi de $36 \%$ entre os pélvicos e de $5 \%$ entre os cefálicos. É de se salientar que existe tendência entre os obstetras de serem mais liberais na indicação de cesárea, quando a apresentação é pélvica.

A ocorrência de partos vaginais $(63,8 \%$ entre os pélvicos) esteve associada à depressão fetal grave no primeiro minuto (índice de Apgar inferior a 4) em 14\% dos casos e à depressão moderada (índice de Apgar entre 4 e 7) em 17,5\%. Em $68,5 \%$ dos casos o índice de Apgar foi igual ou superior a 7 .

Fawole et al. ${ }^{9}$ referiram incidência de parto abdominal de $46,1 \%$, ao passo que Aquino et al. ${ }^{16}$ citaram taxa de parto abdominal de $81 \%$, a despeito da ocorrência de 25,4\% de índice de Apgar inferior a 7 no primeiro minuto. Sabe-se que a apresentação por si só associa-se a maior número de fetos deprimidos, o que justificaria os resultados aqui encontrados.

É importante notar que no quinto minuto houve recuperação quase que total dos conceptos de ambos os grupos; provavelmente, o atendimen- to adequado ao recém-nascido na sala de parto possa ter sido fator determinante para esse fato.

As taxas relacionadas à necessidade de cuidados em ambiente de intensivismo não mostraram relevância $(12,1 \%$ entre os pélvicos), apresentando-se dentro dos níveis normais para o Serviço, considerado de referência para gestações de alto risco na região nordeste do estado. O mesmo pode-se depreender da taxa de mortalidade neonatal $(1,7 \%$ entre os pélvicos) e índices de pequenos $(5,2 \%)$ e grandes para a idade gestacional (10,3\%).

Lindqvist et al. ${ }^{5}$, na Suécia, referiram taxa de mortalidade neonatal de 0,09\%o em grupo de conceptos em apresentação pélvica e paridos pela via vaginal. Meye et al. ${ }^{17}$, no Gabão, ao compararem grupos de partos pélvicos e cefálicos, em gestações iguais ou superiores a 32 semanas, referiram que a taxa de mortalidade neonatal foi de $10,8 \%$, cinco vezes maior que no grupo cefálico (2\%). A disparidade entre esses números torna-se evidente às custas do nível socioeconômico entre nações desenvolvidas e aquelas subdesenvolvidas ou em desenvolvimento.

Ismail et al. ${ }^{18}$ referiram que os resultados perinatais adversos observados em sua série de partos pélvicos estão principalmente relacionados a fetos de muito baixo peso ao nascer, portadores de malformações congênitas e pré-termo, situações de relevo excluídas de nossa pesquisa.

Do presente estudo pode-se concluir que, em casos de apresentação pélvica em gestantes de termo, com pelo menos um parto vaginal anterior, o peso do recém-nascido, a via de parto e o índice de Apgar de primeiro minuto, quando comparados a fetos em apresentação cefálica de gestantes com as mesmas características, apresentaram diferenças significantes. Paridade, idade materna, idade gestacional, tempo de trabalho de parto, índice de Apgar de quinto minuto, necessidade de cuidados em ambiente de intensivismo, incidência de pequenos e grandes para a idade gestacional e taxa de neomortalidade precoce não apresentaram diferenças significantes.

É de se salientar que, a despeito dos resultados benéficos da operação cesariana para partos pélvicos observados no estudo multicêntrico de Hannah et $a .^{3}$, não julgamos como definitiva a indicação da via alta extensiva a todos os casos em que o feto esteja em apresentação pélvica. Sob critérios de seleção rígidos, como os aqui relatados, e conduzido por equipe experiente, o parto vaginal pode ser alternativa segura para esse tipo de apresentação. Importante frisar que no estudo da UMP/HG, as características fetais e maternas, consideradas ideais para a tentativa de parto 
pélvico pela via vaginal, foram identificadas com pequena margem de erro por adestrado grupo de médicos obstetras. Acreditamos, também, que as indicações de cesárea devam ser ampliadas sempre que a probabilidade de nascimento de feto em condições adversas possa ser prevista.

Diferentemente do importante trial desenvolvido por Hannah et al. ${ }^{3}$, quando foram comparados dois grupos de fetos em apresentação pélvica, nascidos pela via abdominal e pela via vaginal, comparamos casos de fetos em apresentação pélvica e em apresentação cefálica, ambos sob condições semelhantes. Julgamos que os fetos em apresentação cefálica, selecionados para o grupo comparativo, seriam portadores das máximas condições de parturição pela via vaginal, fato que pode ser comprovado pelo baixíssima taxa de parto cesáreo (5\%) .

Recentemente Papp $^{19}$ referiu que o parto vaginal deve ser o escolhido, se os seguintes critérios são observados: apresentação pélvica completa, peso fetal estimado entre 2.500 e $3.500 \mathrm{~g}$, adequada pelvimetria sem hiperextensão da cabeça fetal, progressão adequada do trabalho de parto, nenhuma evidência de hipoxia fetal, monitoragem fetal contínua e peso materno inferior a $90 \mathrm{~kg}$. Se esses critérios não puderem ser identificados, tornar-se-á prudente a ultimação do parto pela via abdominal.

Finalmente, a despeito dos números apresentados, reputamos ser necessário acrescentar observações obtidas de forma prospectiva sobre o tema.

\section{ABSTRACT}

Purpose: to assess the obstetric and perinatal outcomes in cases of term newborns in breech presentation, in patients with previous vaginal deliveries, comparing them to term newborns in vertex presentation.

Methods: 8,350 deliveries retrospectively from March 1998 to July 2003 were analysed. Of 419 deliveries (5.1\%) in breech presentation, 58 cases were selected for the study (breech group), according to the following criteria: patients who had had one or more babies through vaginal delivery, gestational age $\geq 37$ weeks, no fetal malformation, no complications in the current pregnancy, birth weight between 2,500 and 3,750 g, and no previous cesarean section. The breech group was matched to 1,327 newborns in vertex position from pregnant women with no previous cesarean section (vertex group). Maternal age, parity, gestational age, delivery way, birth weight, meconiumstained amniotic fluid, 1- and 5-min Apgar score, need of neonatal intensive care unit, and small-and big-for- gestational age newborns were analyzed. Statistical analysis was performed by the $\chi^{2}$ test and by Student's $\mathrm{t}$ test, with the level of significance set at $p<0.05$.

Results: when breech and vertex groups were compared, they showed significant differences regarding the following variables: birth weight $(3,091 \pm 538 \mathrm{~g}$ vs 3,250 $4497 \mathrm{~g}$; $p<0.01$ ), vaginal delivery (63.8 vs $95.0 \% ; p<0.0001)$, cesarean section (36.2 vs 5.0\%; $p<0,0001$ ), and 1-min Apgar score $(p<0.0001)$, respectively.

Conclusions: we conclude that in term fetuses in breech position from pregnant women with previous vaginal deliveries, birth weight, delivery way, and 1-min Apgar score were different compared to fetuses in vertex position from women with the same characteristics.

KEYWORDS: Breech delivery. Perinatal morbidity. Vaginal delivery. Cesarean section.

\section{Referências}

1. Bertini AM, Taborda W. Apresentações anômalas. In: Rezende J, editor. Obstetrícia. 9a ed. Rio de Janeiro: Guanabara Koogan; 2002. p. 1076-88.

2. Cunninghan FG, MacDonald PC, Gant NF, et al. Dystocia due to abnormalities in presentation, position or development of the fetus. In: Williams Obstetrics. 19 ${ }^{\mathrm{a}}$ ed. East Norwalk: Appleton \& Lange, 1993. p. 493-520.

3. Hannah ME, Hannah WJ, Hewson SA, Hodnett ED, Saigal S, Willan AR. Planned caesarean section versus planned vaginal birth for breech presentation at term: a randomized multicentre trial. Lancet 2000; 356: 1375-83.

4. Hannah WJ. The Canadian Consensus on Breech Management at Term. J SOGC 1994; 16:1839-48.

5. Lindqvist A, Norden-Lindeberg S, Hanson U. Perinatal mortality and route of delivery in term breech presentations. Br J Obstet Gynaecol 1997; 104:1288-91.

6. Cheng M, Hannah ME. Breech delivery at term: a critical review of the literature. Obstet Gynecol 1993; 82:605-18.

7. Battaglia FC, Lubchenco LO. A practical classification of newborn infants by weight and gestational age. J Pediatr 1967; 71:159-63.

8. Sobande AA, Archibong EI, Abdelmoneim I, Albar HM. Changing patterns in the management and outcome of breech presentation over a 7-year period. Review from a referral hospital in Saudi Arabia. J Obstet Gynaecol 2003; 23:34-7.

9. Fawole AO, Adeyemi AS, Adewole IF, Omigbodum AO. A ten-year review of breech deliveries at Ibadan. Afr J Med Med Sci 2001; 30:87-90. 
10. Madi JM, Morais EN, Locatelli E, Madi SRC, Rombaldi RL. Síndrome de aspiração do mecônio: análise dos resultados obstétricos e perinatais. Rev Bras Ginecol Obstet 2003; 25:123-8.

11. Morais EN, Porto BS, Weinman AR, et al. Meconium aspiration syndrome and fetal acid-base status at birth. In: Cabero L, Carrera JM, editors. Perinatology 2001. Roma: Monduzzi; 2001. p. 409-12.

12. Morais EN. Mecônio no líquido amniótico. In: Morais EN, Mauad Filho F, editores. Medicina Materna e Perinatal. $1^{\text {a }}$ ed. Rio de Janeiro: Revinter; 2000. p. 207-16.

13. Gonzalez de Dios J, Moya Benavent M, Barbal Rodoreda A, et al. Neonatal morbidity associated with meconial amniotic fluid. An Esp Pediatr 1998; 48:54-9.

14.Tunde-Byass MO, Hannah ME. Breech vaginal delivery at or near term. Semin Perinatol 2003; 27:34-45.
15.Madi JM, Rombaldi RL, Morais EN, et al. Vaginal birth likelihood after one or more previous cesarean section. Int J Gynaecol Obstet 2003; 83 (Suppl 3):40.

16.Aquino MMA, Garcia GM, Rodrigues TMC, et al. Conduta obstétrica na apresentação pélvica. Rev Bras Ginecol Obstet 2000; 22:519-23.

17. Meye JF, Mayi S, Zue AS, Engongah-Beka T, Kendjo $E$, Ole BS. Neonatal prognosis for breech infants delivered vaginally at the Josephine Bongo Maternity Hospital in Libreville, Gabon. Santé 2003; 13:81-4.

18.Ismail MA, Nagib N, Ismail T, Cibils LA. Comparison of vaginal and cesarean section delivery for fetuses in breech presentation. J Perinat Med 1999; 27:339-51.

19.Papp Z. Breech deliveries and cesarean section. J Perinat Med 2003; 31:415-9.

Recebido em: 9/3/2004 Aceito com modificações em: 17/11/2004

\section{Congresso Latinoamericano de Obstetrícia e Ginecologia \\ 15 a 21 de maio de 2005 \\ República Dominicana \\ Realização: FLASOG \\ Informações: \\ Tel: (809) 689-7672 - Fax: (809) 689-7673 \\ E-mail: cteorganizador@flasog2005.com \\ Home Page: www.flasog2005.com}

